07,01

\title{
Влияние зон Гинье-Престона на концентрационную зависимость предела текучести состаренных двухкомпонентных сплавов в условиях высокоскоростной деформации
}

\section{(C) В.В. Малашенко}

Донецкий фризико-технический институт им. А.А. Галкина, Донецк, Украина

Донецкий национальный университет,

Донецк, Украина

E-mail: malashenko@fti.dn.ua

Поступила в Редакцию 21 мая 2019 г.

В окончательной редакции 21 мая 2019 г.

Принята к публикации 30 мая 2019 г.

Теоретически проанализировано скольжение ансамбля краевых дислокаций при высокоскоростной деформации состаренного бинарного металлического сплава. Предел текучести сплава является немонотонной функцией концентрации второго компонента, при определенных условиях имеющей максимум и минимум. Максимум соответствует переходу от доминирующего влияния коллективного взаимодействия дислокаций на формирование спектральной щели к доминированию влияния коллективного взаимодействия атомов второго компонента. Минимум полученной кривой соотвествует переходу от доминирования торможения (drag) дислокации зонами Гинье-Престона (Guinier-Preston zones) к доминированию торможения атомами второго компонента.

Ключевые слова: дислокации, деформация, сплавы, зоны Гинье-Престона.

DOI: 10.21883 /FTT.2019.10.48258.485

Двухкомпонентные металлические сплавы являются важным функциональным материалом, широко используемым в различных отраслях промышленности [1]. В процессе обработки и эксплуатации они могут подвергаться высокоскоростной деформации [2]. Это происходит, в частности, при использовании сварки взрывом, при ударно-волновом воздействии, при воздействии на кристаллы лазерными импульсами высокой мощности, при использовании метода динамического канального углового прессования и при высокоскоростной обработке [3-10]. При этом скорость пластической деформации достигает значений $10^{3}-10^{9} \mathrm{~s}^{-1}[2,7]$, а дислокации движутся со скоростями $v \geq 10^{-2}$ с, где $c-$ скорость распространения поперечных звуковых волн в кристалле, преодолевая встречающиеся потенциальные барьеры без помощи тепловых флуктуаций. Это так называемая динамическая область скоростей.

Существенное влияние на движение дислокаций, а, следовательно, и на механические свойства кристаллов, оказывает динамическое взаимодействие дислокаций с другими дефектами кристаллической структуры, в частности, с атомами второго компонента в двухкомпонентных сплавах и зонами Гинье-Престона, образующимися в этих сплавах на первой стадии старения [11].

При анализе влияния зон Гинье-Престона на динамику дислокаций в сплавах обычно используется метод молекулярной динамики [11-14]. В упомянутых работах концентрационная зависимость механических свойств от концентрации атомов легирующих добавок получена не была. В работе [15] аналитически исследовалось движение ансамбля краевых дислокаций в алюминиевомедном сплаве в условиях высокоскоростной деформации. Исследуемый в этой работе механизм диссипации заключался в необратимом переходе кинетической энергии поступательного движения дислокации в энергию ее изгибных колебаний в плоскости скольжения, которые возникали при взаимодействии дислокаций с зонами Гинье-Престона и атомами меди. Как следует из теории динамического взаимодействия дислокаций со структурными дефектами [16-20], динамика дислокаций при таком механизме диссипации зависит от вида спектра дислокационных колебаний, в первую очередь от наличия щели в дислокационном спектре. Главную роль в рассматриваемой задаче играют коллективное взаимодействие растворенных атомов с дислокацией и коллективное взаимодействие остальных дислокаций ансамбля с этой дислокацией. Конкуренция этих взаимодействий определяет и формирование спектральной щели, и величину силы динамического торможения дислокаций. Сила этих взаимодействий зависит главным образом от концентрации растворенных атомов и плотности дислокаций. В работе [15] исследовался случай доминирующего влияния коллективного взаимодействия атомов меди с дислокацией на формирование ее колебательного спектра. Было показано, что при определенных условиях зависимость предела текучести сплава от концентрации атомов меди становится немонотонной и имеет минимум. В настоящей работе рассмотрен 
как случай доминирующего влияния точечных дефектов на формирование спектральной щели, так и случай доминирования влияния дислокаций ансамбля.

Пусть бесконечные краевые дислокации совершают скольжение под действием постоянного внешнего напряжения $\sigma_{0}$ в положительном направлении оси $O X$ с постоянной скоростью $v$ в кристалле, содержащем зоны Гинье-Престона и атомы второго компонента (рис. 1). Линии дислокаций параллельны оси $O Z$, их векторы Бюргерса $\mathbf{b}=(b, 0,0)$ одинаковы и параллельны оси $O X$. Плоскость скольжения дислокаций совпадает с плоскостью XOZ. Положение $k$-ой дислокации определяется функцией

$$
X_{k}(y=0, z, t)=v t+w_{k}(y=0, z, t) .
$$

Здесь $w_{k}(y=0, z, t)$ - случайная величина, описывающая изгибные колебания дислокации, возбужденные ее взаимодействием с хаотически распределенными дефектами. Среднее значение этой величины по длине дислокации и по хаотическому распределению дефектов равно нулю.

Плоскости зон Гинье-Престона параллельны плоскости скольжения дислокаций, а их центры распределены в кристалле случайным образом. Для простоты все зоны будем считать одинаковыми, то есть имеющими одинаковые радиусы $R$, одинаковую толщину равную диаметру атома второго компонента и одинаковые векторы Бюргерса $\mathbf{b}_{0}=\left(0,-b_{0}, 0\right)$ параллельные оси $O Y$.

Уравнение движения $k$-ой дислокации может быть представлено в следующем виде:

$$
m\left\{\frac{\partial^{2} X}{\partial t^{2}}-c^{2} \frac{\partial^{2} X}{\partial z^{2}}\right\}=b\left[\sigma_{0}+\sigma_{x y}^{d}+\sigma_{x y}^{\mathrm{G}}\right]+F_{k}-B \frac{\partial X}{\partial t} .
$$

где $\sigma_{x y}^{d}-$ компонента тензора напряжений, создаваемых точечными дефектами на линии дислокации, $\sigma_{x y}^{\mathrm{G}}-$ компонента тензора напряжений, создаваемых на этой линии зонами Гинье-Престона, $F_{k}-$ сила, действующая на дислокацию со стороны остальных дислокаций ансамбля, $m$ - масса единицы длины дислокации (массы всех дислокаций считаем одинаковыми), $c$ - скорость распространения в кристалле поперечных звуковых волн, $B$ - константа демпфирования, обусловленная фононными, магнонными или электронными механизмами диссипации. Здесь, как и в работах [15-20], будем считать выполненным условие $\left[B b v /\left(m c^{2}\right)\right] \ll 1$, позволяющее пренебречь влиянием константы $B$ на силу торможения дислокации структурными дефектами.

Полная сила торможения дислокации будет равна

$$
F=F_{\mathrm{G}}+\mathrm{G}_{d}+B v .
$$

Здесь $F_{\mathrm{G}}-$ сила торможения (drag) дислокации зонами Гинье-Престона, $F_{d}-$ сила торможения, обусловленная взаимодействием дислокации с атомами второго компонента, $B v-$ фононное торможение.

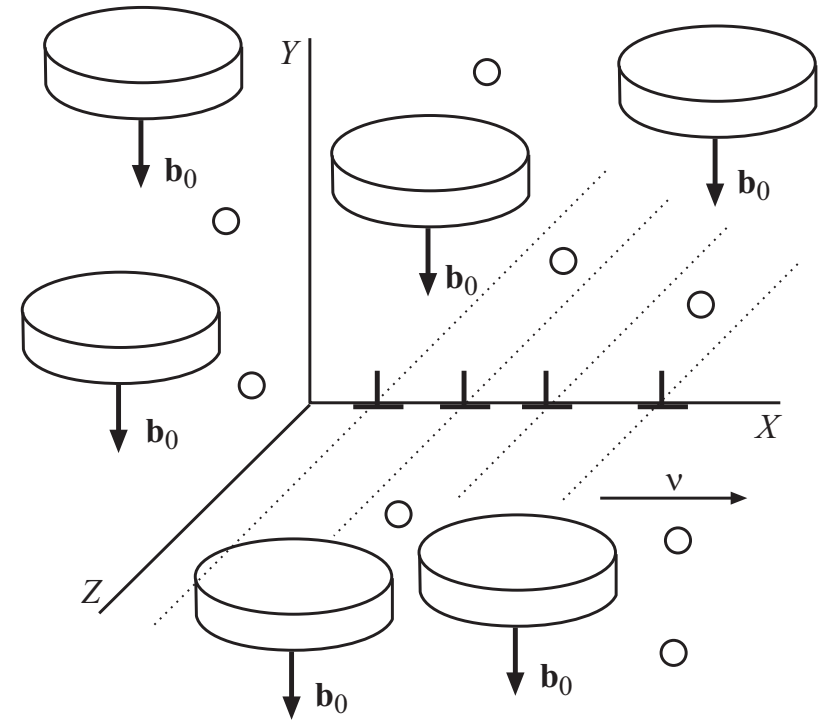

Рис. 1. Скольжение ансамбля краевых дислокаций в состаренном бинарном сплаве, содержащем зоны Гинье-Престона.

Сила динамического торможения движущейся краевой дислокации структурными дефектами согласно [15-20], может быть вычислена по формуле

$$
F_{d e f}=\frac{n b^{2}}{8 \pi^{2} m} \int d^{3} q\left|q_{x}\right|\left|\sigma_{x y}(\mathbf{q})\right|^{2} \delta\left(q_{x}^{2} v^{2}-\omega^{2}\left(q_{z}\right)\right),
$$

где $\omega\left(q_{z}\right)$ - спектр дислокационных колебаний, $\sigma_{x y}(\mathbf{q})-$ Фурье-образ компоненты тензора напряжений, созданных соответствующим дефектом (в нашем случае это либо точечный дефект, либо зона Гинье-Престона), $n$ - объемная концентрация соответствующих дефектов.

В рассматриваемом нами случае спектр дислокационных колебаний имеет вид

$$
\omega^{2}\left(q_{z}\right)=c^{2} q_{z}^{2}+\Delta^{2} .
$$

Щель $\Delta$ в колебательном спектре может возникать благодаря коллективному взаимодействию растворенных атомов с дислокацией и, согласно [15], описывается формулой

$$
\Delta=\Delta_{d}=\frac{c}{b}\left(n_{d} \chi^{2}\right)^{1 / 4}
$$

где $n_{d}$ - безразмерная концентрация растворенных атомов, $\chi$ - параметр их размерного несоответствия.

Взаимодействие движущихся дислокаций ансамбля также способно влиять на формирование колебательного спектра. Вклад этого взаимодействия в величину щели согласно [19] определяется формулой

$$
\Delta_{d i s}=\pi b \sqrt{\frac{\mu \rho}{6 \pi m(1-\gamma)}} \approx c \sqrt{\rho},
$$

где $\rho$ - плотность подвижных дислокаций, $\mu-$ модуль сдвига, $\gamma$ - коэффициент Пуассона. 
Как следует из работы [15], сила динамического торможения дислокации зонами Гинье-Престона имеет характер сухого трения при выполнении условия $v<v_{L}=R \Delta$. В нашем случае это условие приобретает следующий вид:

$$
v<v_{L}=c \sqrt{\rho b^{2}\left(1+\sqrt{n_{d} / n_{1}}\right)} ; \quad n_{1}=\left(\frac{\rho b^{2}}{\chi}\right)^{2} .
$$

После выполнения необходимых вычислений получим выражение для силы торможения дислокации зонами Гинье-Престона в виде

$$
F_{\mathrm{G}}=\frac{n_{\mathrm{G}} \mu b_{0}^{2} R}{\sqrt{\rho\left(1+\sqrt{n_{d} / n_{1}}\right)}} .
$$

Здесь $n_{\mathrm{G}}-$ объемная концентрация зон ГиньеПрестона.

Аналогичным образом можем получить выражение для силы динамического торможения дислокации атомами второго компонента

$$
F_{d}=\frac{n_{d} \mu \chi^{2}}{b \rho\left(1+\sqrt{n_{d} / n_{1}}\right)} \frac{v}{c} .
$$

Выполним численные оценки. Для значений $\rho=10^{15} \mathrm{~m}^{-2}, b=3 \cdot 10^{-10} \mathrm{~m}, c=3 \cdot 10^{3} \mathrm{~m} / \mathrm{s}, a=100 b$ получим $v_{L}=c$, для $a=10 \mathrm{~b}$ соответственно $v_{L}=10^{-1} \mathrm{c}$. Полученные значения попадают в исследуемый нами динамический интервал скоростей.

Зная силу динамического торможения дислокации, мы можем получить выражение для динамического предела текучести бинарных сплавов

$$
\tau=\frac{\eta}{\sqrt{\left(1+\sqrt{n_{d} / n_{1}}\right)}}+\frac{\beta n_{d}}{\left(1+\sqrt{n_{d} / n_{1}}\right)}+\lambda .
$$

Здесь введены обозначения

$$
\beta=\frac{\mu \chi^{2} \dot{\varepsilon}}{\rho^{2} b^{3} c}, \quad \eta=\frac{n_{\mathrm{G}} \mu b_{0} R}{\sqrt{\rho}}, \quad \beta=\frac{B \dot{\varepsilon}}{\rho b c},
$$

где $\dot{\varepsilon}-$ скорость пластической деформации.

Динамический предел текучести является немонотонной функцией концентрации атомов второго компонента и имеет максимум при $n_{d}=n_{1}$ и минимум при $n_{d}=n_{2}$

$$
n_{2}=\sqrt[3]{\left(\frac{n_{\mathrm{G}} b^{3} R \rho c}{\dot{\varepsilon} \chi^{3 / 2}}\right)^{4}}
$$

Максимум соответствует переходу от доминирующего влияния коллективного взаимодействия дислокаций на формирование спектральной щели к доминированию влияния коллективного взаимодействия атомов второго компонента. Минимум полученной кривой соотвествует переходу от доминирования торможения дислокации зонами Гинье-Престона к доминированию торможения атомами второго компонента.

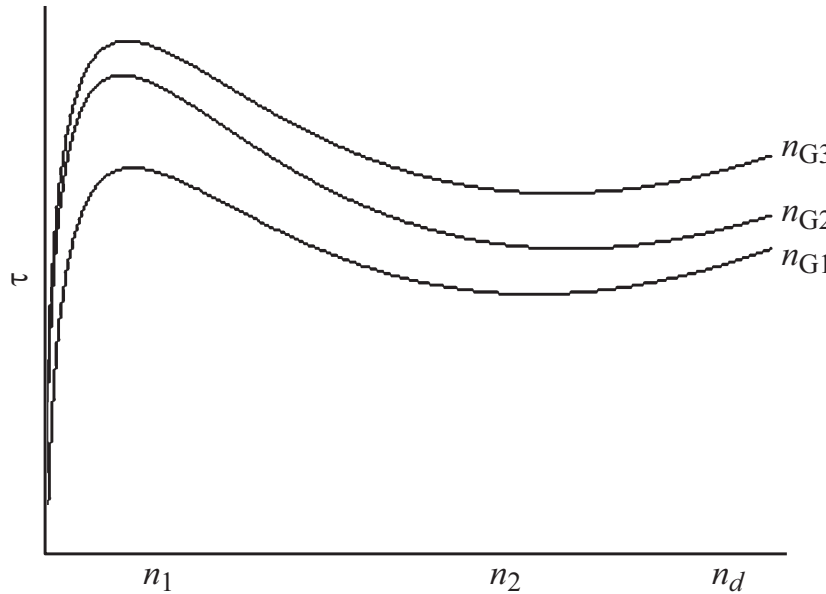

Рис. 2. Зависимость динамического предела текучести бинарного сплава от концентрации атомов второго компонента при разных значениях концентрации зон Гинье-Престона $\left(n_{\mathrm{G} 3}>n_{\mathrm{G} 2}>n_{\mathrm{G} 1}\right)$.

Выполним численные оценки. Для значений $b=4 \cdot 10^{-10} \mathrm{~m}, \quad \gamma=0.3, \quad \chi=10^{-1}, \quad R=10 b$, $n_{\mathrm{G}}=10^{23}-10^{24} \mathrm{~m}^{-3}, \quad \rho=6 \cdot 10^{15} \mathrm{~m}^{-2}, \quad c=3 \cdot 10^{3} \mathrm{~m} / \mathrm{s}$, $\dot{\varepsilon}=10^{6} \mathrm{~s}^{-1}$ получим $n_{1}=10^{-3}-10^{-4}$ и $n_{2}=10^{-1}-10^{-2}$. Зависимость динамического предела текучести от концентрации атомов второго компонента приведена на рис. 2.

С ростом концентрации зон Гинье-Престона положение минимума смещается в сторону больших значений концентрации атомов второго компонента, положение максимума при этом не изменяется.

Полученные результаты могут быть полезны при анализе высокоскоростной деформации сплавов.

\section{Список литературы}

[1] P.N. Mayer, A.E. Mayer. J. Appl. Phys. 120, 075901 (2016).

[2] J. Lee, D. Veysset, J. Singer, M. Retsch, G. Saini, T. Pezeril, K. Nelson, E. Thomas. Nature Commun. 3, 1164 (2012).

[3] Г.А. Малыгин, О.В. Клявин. ФТТ 59, 10. 1964 (2017).

[4] H. Hallberg, K. Ryttberg, M. Ristinmaa. ASCE J. Eng. Mech. 135, 345 (2009).

[5] D. Batani. EPL 114, 6500 (2016).

[6] D. Tramontina, E. Bringa, P. Erhart, J. Hawreliak, T. Germann, R. Ravelo, A. Higginbotham, M. Suggit, J. Wark, N. Park, A. Stukowski, Y. Tang. High Energy Density Phys. 10, 9 (2014).

[7] E.B. Zaretsky, G.I. Kanel. J. Appl. Phys. 114, 083511 (2013).

[8] Г.И. Канель, В.Е. Фортов, С.В. Разоренов. УФН 177, 809 (2007).

[9] И.Н. Бородин, А.Е. Майер. ЖТФ 83, 76 (2013).

[10] В.И. Зельдович, Е.В. Шорохов, С.В. Добаткин, Н.Ю. Фролова, А.Э. Хейфец, И.В. Хомская, П.А. Насонов, А.А. Ушаков. ФММ 111, 439 (2011). 
[11] A.Yu. Stroev, O.I. Gorbatov, Yu.N. Gornostyrev, P.A. Korzhavyi. Phys. Rev. Mater. 2, 033603 (2018).

[12] W. Verestek, A.-P. Prskalo, M. Hummel, P. Binkele, S. Schmauder. Phys. Mesomech. 20, 291 (2017).

[13] А.Ю. Куксин, А.В. Янилкин. МТТ 1, 54 (2015).

[14] A.V. Yanilkin, V.S. Krasnikov, A.Yu. Kuksin, A.E. Mayer. Int. J. Plast. 55, 94 (2014).

[15] В.В. Малашенко. Письма в ЖТФ 44, 47 (2018).

[16] В.В. Малашенко. ФТТ 58, 1973 (2016).

[17] В.В. Малашенко. ФТТ 57, 2388 (2015).

[18] В.В. Малашенко. Письма в ЖТФ 45, 5 (2019).

[19] В.В. Малашенко. ФТТ 56, 1528 (2014).

[20] V.V. Malashenko. Physica B 404, 2, 3890 (2009).

Редактор Т.Н. Василевская 